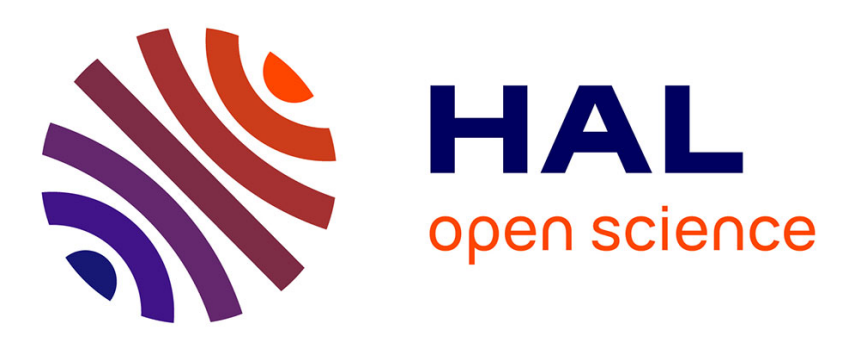

\title{
Tunable source of infrared pulses in gas-filled hollow core capillary
}

Olivia Zurita-Miranda, Coralie Fourcade-Dutin, Pierre Béjot, Frederic Fauquet, Jean-Paul Guillet, Frédéric Darracq, Patrick Mounaix, Hervé Maillotte, Damien Bigourd

\section{To cite this version:}

Olivia Zurita-Miranda, Coralie Fourcade-Dutin, Pierre Béjot, Frederic Fauquet, Jean-Paul Guillet, et al.. Tunable source of infrared pulses in gas-filled hollow core capillary. Laser Congress (virtual event), OSA, Oct 2020, San Diego, France. hal-02965842

\section{HAL Id: hal-02965842 \\ https://hal.science/hal-02965842}

Submitted on 13 Oct 2020

HAL is a multi-disciplinary open access archive for the deposit and dissemination of scientific research documents, whether they are published or not. The documents may come from teaching and research institutions in France or abroad, or from public or private research centers.
L'archive ouverte pluridisciplinaire HAL, est destinée au dépôt et à la diffusion de documents scientifiques de niveau recherche, publiés ou non, émanant des établissements d'enseignement et de recherche français ou étrangers, des laboratoires publics ou privés. 


\title{
Tunable source of infrared pulses in gas-filled hollow core capillary
}

\author{
Olivia Zurita-Miranda ${ }^{1,2}$, Coralie Fourcade-Dutin ${ }^{2}$, Pierre Béjot $^{3}$, Frederic Fauquet $^{2}$, Jean-Paul Guillet ${ }^{2}$, \\ Frederic Darracq ${ }^{2}$, Patrick Mounaix ${ }^{3}$, Hervé Maillotte ${ }^{1}$, Damien Bigourd ${ }^{1,2}$ \\ ${ }^{1}$ Femto-st, Département d'Optique, UMR6174, Université Bourgogne Franche-Comté,25030 Besançon, France \\ ${ }^{2}$ Laboratoire IMS, UMR CNRS 5218, Université de Bordeaux, 33405 Talence, France \\ ${ }^{3}$ Laboratoire Interdisciplinaire Carnot de Bourgogne UMR CNRS 6303, Université Franche-Comté, 2178 Dijon, France \\ olivia.zurita-miranda@u-bordeaux.fr
}

\begin{abstract}
We report a tunable source that generates pulses in the infrared from an optical parametric amplification in a gas-filled hollow core capillary based on four-wave mixing process, in which the phase matching strongly depends on the gas pressure and the pump. In our case, we have generated pulses from 1 to $1.6 \mu \mathrm{m}$ in the sub- $\mu \mathrm{J}$ level together with a parametric amplification in the visible.
\end{abstract}

\section{Introduction}

Since these recent years, an interest is growing for new generator in the ultra-short pulse regime in the near-infrared with the capability to reach the mid-infrared for demanding applications as life and molecular sciences or lasermatter interaction experiment [1]. These pulses are often generated from a laser emission or nonlinear photonic processes to generate a down converted frequency. However, the energy of the incoming laser pulse can reach the limit imposed by an optical damage, thus strongly compromise the energy scalability. In addition, the spectral window and the tunability are limited by the transparency material [2]. Gas-filled hollow core capillary (HCC) brings several benefits from solid materials as a higher damage threshold. In addition, some configurations are very interesting to get low losses in the fundamental mode or to excite high-order modes to reach other phase matched processes. The gas pressure can be used as a tunable parameter since it modifies directly the nonlinearity and the group velocity dispersion. Therefore, four wave mixing (FWM) based parametric process in a HCC is a key process to enable the creation of ultra-fast sources with a very large tunability. For example, a source in the visible has been implemented at a level of $10 \mu \mathrm{J}$ [3] and in the near infrared at $1.4 \mu \mathrm{m}$ with an energy of $5 \mu \mathrm{J}$ and a duration of 45 fs [4]. On the other hand, deep-ultraviolet ultrashort pulses have been generated using four-wave mixing in gas-filled Kagomé photonic crystal fiber with a conversion efficiency of 38\% [5]. Kida at al. [6] have been used FWM in combination with (OPA) in HCC to amplify a supercontinuum signal, with a amplification gain up to 100 times. In this work, we highlight the tunability of the FWM based source in the infrared from $1 \mu \mathrm{m}$ to $1.6 \mu \mathrm{m}$ together with a parametric amplification of a continuum in the visible.

\section{Experimental arrangement and condition}

The degenerated FWM is a process involving two optical fields at different frequencies to produce a third wave in a third-order nonlinearity medium. In the following, we consider one pump pulse and a signal (at the angular frequency $\omega_{1}$ and $\omega_{2}$ respectively), that co-propagate in the HCC. When a phase matching condition is achieved, part of the pump energy is transferred to the signal and create a new component at $\omega_{3}$; the idler, according to the energy conservation relation, $\omega_{3}=2 \omega_{1}-\omega_{2}$. We firstly calculated the linear phase matching, given by $2 \beta\left(\omega_{1}\right)-$ $\beta\left(\omega_{2}\right)-\beta\left(\omega_{3}\right)$ with $\beta$ is the wave vector that depends on the waveguide and the gas for our experimental condition; i.e a Argon filled capillary with a core diameter of $150 \mu \mathrm{m}$. Fig. 1.a shows that a phase matching condition can be obtained in the near infrared and the visible when the pressure is adjusted between 1 and 5 bar for a pump wavelength of $800 \mathrm{~nm}$. The experimental set-up is displayed in Fig. 1. The pump pulses are directly provided by a $1 \mathrm{kHz}$ Ti:Sappire (Ti:Sa) laser source ( $800 \mathrm{~nm}, 120 \mathrm{fs})$ in combination with a continuum signal $(420-600 \mathrm{~nm})$. This signal is generated from the remaining part of the pump pulse focused into a 5-mm thick sapphire plate. The two collinear beams were coupled in the $30 \mathrm{~cm}$ long HCC. Their relative delay was controlled by an optical delay line endowed with a translation stage. The HCC is place inside a chamber with two 5-mm-thick windows and filled it with Argon. At the output chamber, the beam was divided in the visible and infrared, and detected by specific spectrometers simultaneously. 

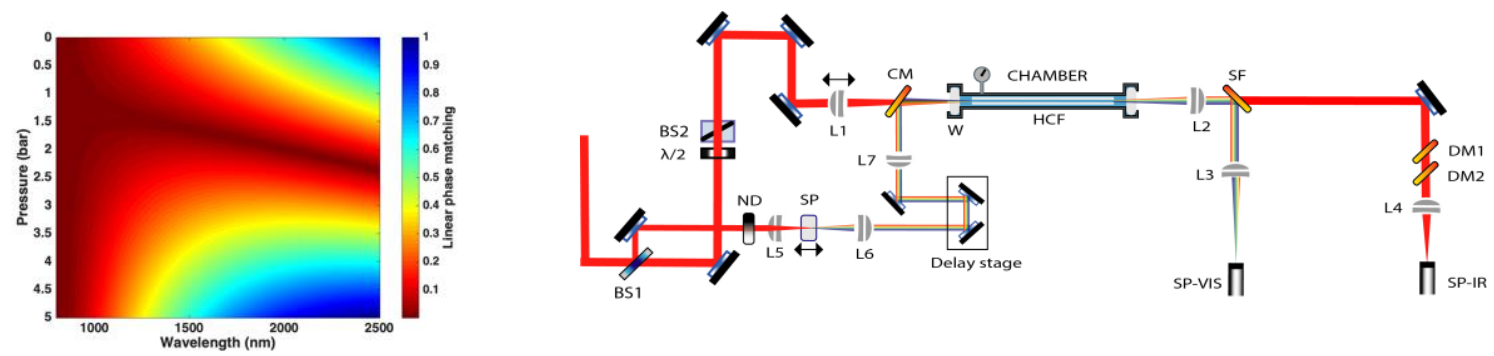

Fig. 1 a) Linear phase matching in HCC core diameter of $150 \mu \mathrm{m}$ filled argon, pump energy $150 \mu \mathrm{J}$ at $120 \mathrm{fs}$. b) Experimental configuration. BS, beam splitter; MR mirror; ND neutral density filter; L lens; CM, Cold mirror cutoff 700 nm; SP sapphire plate; SF short-pass filter, DM dichroic mirror, SP-VIS Visible Optics Spectrometer, SP-IR Infra-red Spectrometer.

\section{Results}

When the seed is injected together with the pump pulse in the Argon filled HCC for a pump energy of $128 \mu \mathrm{J}$ and a pressure of 4 bar, the visible spectrum is amplified with a gain of 80 (Fig. 2.a). Due to the chirp imposed by the continuum, the tunability is achieved with the delay stage to overlap the targeted part of the seed with the pump. In addition to the seed amplification, pulses in the near infrared are generated and tuned from 1 to $1.6 \mu \mathrm{m}$ (Fig. $2 . \mathrm{b}$ ). The stronger generated pulses is at $\sim 1.5 \mu \mathrm{m}$ corresponding to the strongest amplification in the shorter wavelength part. Simultaneously, the pump acquired self-phase modulation in the capillary, which modified the pulse shape and therefore varied the expected phase matching (Fig.1.a).
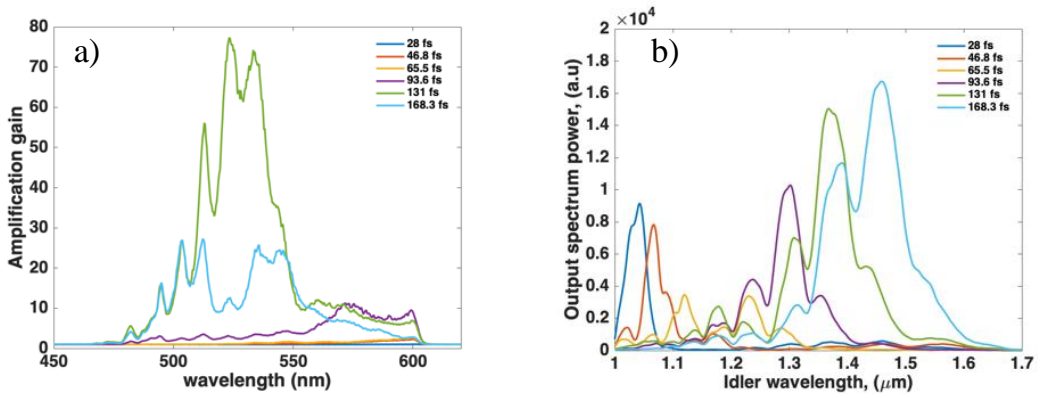

Fig. 2 a) Amplified signal spectra and b) generated infrared idler spectra at different time delays.

In conclusion, we implemented a tunable source in the infrared driven by a FWM based parametric process in a Argon filled HCC. We demonstrated a wide tunability from 1 to $1.6 \mu \mathrm{m}$ together with the visible amplification. We are currently working to adjust the optimal conditions, which allow a best conversion efficiency and a wider tunability toward the mid-infrared by adjusting the pump pulse as [7] and gas characteristic.

\section{References}

[1]B. Wolter et al., "Strong-field physics with Mid-IR fields," PhysRevX 5, 021034-16 (2015).

[2]J. C. Travers, W. Chang, J. Nold, N. Y. Joly, and P. St. J. Russell, "Ultrafast nonlinear optics in gas-filled hollow-core photonic crystal fibers," JOSA B 28, A11-26 (2011).

[3]A. G. Ciriolo et al., "Generation of ultrashort pulses by four wave mixing in a gas-filled hollow core fiber," JO. 20, 125503 (2018)

[4]D. Faccio, A. Grün, P. K. Bates, O. Chalus, and J. Biegert, “Optical amplification in the near-infrared in gas-filled hollow-core fibers,”OL 34, 2918-2920 (2009).

[5] F. Belli, A. Abdolvand, J. C. Travers, P. J. Russell, "Highly efficient deep UV generation by four-wave mixing in gas-filled hollow-core photonic crystal fiber", Opt. Lett. 44, 5509-5512 (2019)

[6] Y. Kida, T. Imasaka, "Optical parametric amplification of a supercontinuum in a gas" APB. 116, 673-680 (2014)

[7] O. Vanvincq, C. Fourcade-Dutin, A. Mussot, E. Hugonnot, D. Bigourd, "Ultrabroadband fiber optical parametric amplifiers pumped by chirped pulses. Part 1: analytical model," JOSA B 32, 1479-1487 (2015) 